\title{
Veterinary Research reviewer acknowledgement 2014
}

Michel Brémont ${ }^{*}$ and Élodie Coulamy

\section{Contributing reviewers}

The Veterinary Research editorial team would sincerely like to thank all of our reviewers who contributed to peer review for the journal in 2014.

\author{
E.M. Abdelwhab \\ Germany
}

Mathias Ackermann

Switzerland

Alexandra Adams

United Kingdom

Alvaro Aguilar Setién

Mexico

Henri Agut

France

Raul Almeida

United States of America

Sonia Almeria

Spain

Irene Alvarez

Argentina

Mathieu Andraud

France

Elisavet Angelidou

Greece

Virginia Aragon

Spain

Clarice Arns

Brazil

\section{Shawn Babiuk}

Canada

\author{
Douwe Bakker \\ Netherlands
}

Mitchell Balish

United States of America

Keith Ballingall

United Kingdom

\section{Herman Barkema}

Canada

Michael Baron

United Kingdom

Walter Basso

Switzerland

Max Bastian

Germany

Andrew Bean

Australia

Douglas Begg

Australia

Graham John Belsham

Denmark

\section{Ana Bento}

United Kingdom

Are Berentsen

United States of America

Luiz Bermudez

United States of America
Jean-Francois Bernardet

France

Kateri Bertran

United States of America

Stephane Biacchesi

France

Stephen Bishop

United Kingdom

Barbara Blacklaws

United Kingdom

Damer Blake

United Kingdom

Sandra Blome

Germany

Rosa Bolea

Spain

Manuel Vitor Borca

United States of America

Thomas Boren

Sweden

Alex Bossers

Netherlands

Annemarie Bouma

Netherlands

Andrew Bowman

United States of America

\footnotetext{
* Correspondence: veterinary.research@jouy.inra.fr

Veterinary Research, Unité de Virologie et Immunologie Moléculaires, INRA,

Domaine de Vilvert, 78352 Jouy-en-Josas cedex, France
} 
Kevin Bown

United Kingdom

Filip Boyen

Belgium

Paulo Brandão

Brazil

Samantha Brandler

France

Volker Brinkmann

Germany

Jennifer Brisbin

Canada

Barbara Brito

United States of America

Collette Britton

United Kingdom

Erin Bromage

United States of America

Glenn Browning

Australia

Raymond Bujdoso

United Kingdom

Christian Burvenich

Belgium

Patrick Butaye

Belgium

Luis Calvinho

Argentina

Franco Cardone

Italy

Alexandre Caron

Zimbabwe

Chanhee Chae

Korea, South

Christophe Chevalier

France

Francesca Chianini

United Kingdom

Koen Chiers

Belgium

Rémi Choquet

France

Shafiqul Chowdhury

United States of America
Christine Citti

France

Axel Cloeckaert

France

Bertrand Collet

United Kingdom

Ellen Collisson

United States of America

Emmanuel Comoy

France

Andrew Conlan

United Kingdom

Timothy Connelley

United Kingdom

Franz Josef Conraths

Germany

Richard Cook

United States of America

Brian Cooke

Australia

Kevin Coombs

Canada

Eric Cox

Belgium

C. P. Binesh

India

Helen Crooke

United Kingdom

Sarah Culloty

Ireland

Cristina Cunha

United States of America

Adam Cunningham

United Kingdom

Charles Czuprynski

United States of America

Rkia Dardari

Canada

Stuart Dashper

Australia

Todd Davis

United States of America

Stephen Davis

Australia
Andrew Davison

United Kingdom

Michael Day

United States of America

Jeroen De Buck

Canada

Henri De Greve

Belgium

Sarne De Vliegher

Belgium

Sjaak de Wit

Netherlands

Lieven De Zutter

Belgium

Annemie Decostere

Belgium

Bernard Delmas

France

Piet Deprez

Belgium

Bert Devrient

Belgium

Jeroen Dewulf

Belgium

Adama Diallo

Austria

Isabelle Dimier-Poisson

France

Steven Djordjevic

Australia

Javier Dominguez

Spain

Charles Martin Dozois

Canada

Richard Ducatelle

Belgium

Mariette Ducatez

France

Christian Ducrot

France

Paul Duff

United Kingdom

Peter David Eckersall

United Kingdom 
Torsten Eckstein

United States of America

Venessa Eeckhaut

Belgium

Susanne Eisenberg

Netherlands

Gary Entrican

United Kingdom

Ronald Erskine

United States of America

Nicolas Eterradossi

France

Øystein Evensen

Norway

James Evermann

United States of America

Claes Fellström

Sweden

Giancarlo Ferrari

Italy

Richard Ferrero

Australia

Thomas Ficht

United States of America

Bram Flahou

Belgium

Eduardo Flores

Brazil

Laurence Flori

France

Anthony Fooks

United Kingdom

Andrew Forbes

United Kingdom

Guillaume Fournié

United Kingdom

Caroline Frey

Switzerland

Joachim Frey

Switzerland

Sarah Gabriël

Belgium

Nellie Gagne

Canada
Barbara Gallagher

Ireland

Jean-Pierre Gangneux

France

Juan J. Garrido

Spain

Joseba M. Garrido

Spain

M Carolyn Gates

United Kingdom

Pierre Germon

France

Wilhelm Gerner

Austria

Laura Gillim-Ross

United States of America

Luis Gabriel Gimenez Lirola

United States of America

Elisabetta Giuffra

France

Liz Glass

United Kingdom

Anja Globig

Germany

Jose L Gonzales

United Kingdom

Marcelo Gottschalk

Canada

Kaare Græsbøll

Denmark

Simon Graham

United Kingdom

Philip Griebel

Canada

Martin H Groschup

Germany

Guiquan Guan

China

Luca Guardabassi

Denmark

Ronald Guenther

Germany

Carlton Gyles

Canada
Freddy Haesebrouck

Belgium

Kim Halpin

Australia

John Hammond

United Kingdom

David Hampson

Australia

Josee Harel

Canada

Balazs Harrach

Hungary

Christopher Heaney

United States of America

Andrew Hemphill

Switzerland

Uri Hershberg

United States of America

Marc Heyndrickx

Belgium

Frederic Hoerr

United States of America

Ole Højberg

Denmark

Jason Holland

United Kingdom

Martin Holland

United Kingdom

Jayne Hope

United Kingdom

Sawako Hori-Oshima

Mexico

David Horohov

United States of America

Emily Hotchkiss

United Kingdom

Jos Houdijk

United Kingdom

Valerie Hughes

United Kingdom

Munir Iqbal

United Kingdom

Naoki Isobe

Japan 


\author{
Mario Jacques \\ Canada \\ Hugh Jones \\ Australia \\ Michael Jones \\ United Kingdom \\ Jorunn B Jørgensen \\ Norway \\ Gregers Jungersen \\ Denmark \\ Ramon A. Juste \\ Spain \\ Pete Kaiser \\ United Kingdom \\ Bernd Kaspers \\ Germany \\ Guenther Keil \\ Germany \\ Clayton Kelling \\ United States of America \\ David Kelton \\ Canada \\ Alyson Kelvin \\ Canada \\ Kathryn Kemper \\ Australia \\ Michael Kent \\ United States of America \\ David Kerr \\ United States of America \\ Anthony Keyburn \\ Australia \\ Hiroshi Kida \\ Japan \\ Donald King \\ United Kingdom \\ Peter Kirkland \\ Australia \\ Eyal Klement \\ Israel \\ Don Klinkenberg \\ Netherlands \\ Wolfgang Koester \\ Canada
}

Michael Kogut

United States of America

Nicholas Komar

United States of America

Peter Krawczel

United States of America

Naveen Kumar
India

Sachin Kumar

India

Gael Kurath

United States of America

Elisabetta Lambertini

United States of America

Benedicte Lambrecht

Belgium

Lucie Lamontagne

Canada

Frederic Lantier

France

Neus Latorre-Margalef

United States of America

Victoria Lawson

Australia

Ghislaine Le Gall-Recule

France

Ronan Le Goffic

France

Yves Le Loir

France

Sophie Le Poder

France

François Lefèvre

France

Alexandre Leitão

Portugal

Caroline Leroux

France

Martin Lessard

Canada

Randall Levings

United States of America

Zejun Li

China
Geneviève Libeau

France

Ann Lindberg

Sweden

John Lippolis

United States of America

Yoram Louzoun

Israel

Shuhong Luo

China

Blanca Lupiani

United States of America

Wenjun Ma

United States of America

Heba Mabrouk-Mostafa

United States of America

Rosangela Zacarias Machado

Brazil

N. James Maclachlan

United States of America

Vanesa Madan

Germany

Lone Madsen

Denmark

Dominiek Maes

Belgium

David Maggs

United States of America

Kathy Magor

Canada

Helena Maier

United Kingdom

Viviana Malirat

Argentina

Francois Malouin

Canada

Daniel Marc

France

An Martel

Belgium

Paolo Martelli

Italy

Ann Martens

Belgium 
Hugh Mason

United States of America

Enric Mateu

Spain

James McGettigan

United States of America

Tom McNeilly

United Kingdom

Douglas Scott Merrell

United States of America

Francois Meurens

Canada

Simon Milling

United Kingdom

F. Chris Minion

United States of America

Rebecca Mitchell

United States of America

Petra Moebius

Germany

Fawzi Mohamed

United States of America

Maria Montoya

United Kingdom

Igor Morozov

United States of America

Ivan Morrison

United Kingdom

Liam Morrison

United Kingdom

Claude P. Muller

Luxembourg

Thomas Mueller

Germany

Claudio Murgia

United Kingdom

Michael Murtaugh

United States of America

Venugopal Nair

United Kingdom

Hans Nauwynck

Belgium

Mirjam Nielen

Netherlands
John Noh

United States of America

Romolo Nonno

Italy

Alejandro Núñez

United Kingdom

Jose I. Núñez

Spain

Norio Ohashi

Japan

Gaetano Oliva

Italy

Marinda Oosthuizen

South Africa

Klaus Osterrieder

United States of America

Tibor Papp

Hungary

Mark Parcells

United States of America

Nola Parsons

South Africa

Frank Pasmans

Belgium

Angela Pearson

Canada

Niels Pedersen

United States of America

Ben Peeters

Netherlands

Andres Perez

United States of America

Denis Pierard

Belgium

Gina Pighetti

United States of America

Patrick Pithua

United States of America

Karren Plain

Australia

Brandon Plattner

Canada

Yves Portejoie

France
Jacob Post

Netherlands

Edoardo Pozio

Italy

Marian Price-Carter

New Zealand

Maureen Purcell

United States of America

Auriol Purdie

Australia

Pascal Rainard

France

Thomas Bruun Rasmussen

Denmark

Johanna Marina Jacoba Rebel

Netherlands

Patrick Redig

United States of America

Robert Reiner

United States of America

Bertus Rima

United Kingdom

Espen Rimstad

Norway

Julian Rood

Australia

Marty Roop

United States of America

Nicolas Rose

France

Mirko Rossi

Finland

Ronan Rouxel

France

Franco Maria Ruggeri

Italy

Till Rümenapf

Germany

Edward Rybicki

South Africa

Ivan Rychlik

Czech Republic

Andrew Rycroft

United Kingdom 
Armin Saalmueller

Austria

Konrad Sachse

Germany

Xavier Saelens

Belgium

Siba Samal

United States of America

Leonhard Schnittger

Argentina

Manuela Schnyder

Switzerland

Ynte Schukken

United States of America

Walter Schulz-Schaeffer

Germany

Manon Schuppers

Switzerland

Chris Secombes

United Kingdom

Luigi Sedda

United Kingdom

Mariela Segura

Canada

Hans-Martin Seyfert

Germany

Awad A. Shehata

Germany

M.S. Shekhar

India

I. Martin Sheldon

United Kingdom

Yujiang Shi

United States of America

Brian Shiels

United Kingdom

Craig Shoemaker

United States of America

Marion M Simmons

United Kingdom

Marek Sinkora

Czech Republic

Anja Sipka

United States of America
Michael Skinner

United Kingdom

Annemieke Smet

Belgium

Adrian Smith

United Kingdom

Kenneth Söderhäll

Sweden

Daesub Song

Korea, South

Stephanie Sonnberg

United States of America

Maria Rathmann Sørensen

Denmark

Marina Spinu

Romania

Tomasz Stadejek

Poland

David Stallknecht

United States of America

Mieke Steensels

Belgium

Mark Stevens

United Kingdom

Norbert Stockhofe-Zurwieden

Netherlands

Anne K Storset

Norway

Tanja Strive

Australia

Artur Summerfield

Switzerland

Lotta-Riina Sundberg

Finland

Gerd Sutter

Germany

Nicholas Svitek

Kenya

Raymond Sweeney

United States of America

Michael Szostak

Austria

Geraldine Taylor

United Kingdom
Gergely Tekes

Germany

Eileen Thacker

United States of America

Etienne Thiry

Belgium

Kim D. Thompson

United Kingdom

Peter Thompson

South Africa

Tijs Tobias

Netherlands

Panagiotis Tourlomousis

United Kingdom

Bryan Troxell

United States of America

Vikram Vakharia

United States of America

Thierry van den Berg

Belgium

Wim van der Hoek

Netherlands

Sylvia van Drunen Littel-van den Hurk Canada

Koenraad Van Hoorde

Belgium

Filip Van Immerseel

Belgium

Kristien Van Reeth

Belgium

Debby van Riel

Netherlands

Vicky van Santen

United States of America

Michael van Straten

Israel

Wannes Vanderhaeghen

Belgium

Alain Vanderplasschen

Belgium

Marian Varady

Slovakia

Eva Vareckova

Slovakia 
Jorge Velasco-Hernandez

Mexico

Elin Verbrugghe

Belgium

Elisabeta Vergu

France

Leen Verhaert

Belgium

Mafalda Viana

United Kingdom

Stefan Vilcek

Slovakia

Vladimir Vrba

Czech Republic

Bettina Wagner

United States of America

Thomas Wahli

Switzerland
Cheryl Waldner

Canada

Bernard Wasinski

Poland

Pierre Wattiau

Belgium

Richard Webby

United States of America

Scott Wells

United States of America

C Jane Welsh

United States of America

Dirk Werling

United Kingdom

Stephen White

United States of America

Gary Whittaker

United States of America
Paul Wigley

United Kingdom

Rebecca Wilkes

United States of America

Robert Wolf

Canada

Yongjun Yu

United States of America

Mark Zabel

United States of America

Jianqiang Zhang

United States of America

Annetta Zintl

Ireland

Siamak Zohari

Sweden 